\title{
Cross-Domain Collaboration for Improved IDS Rule Set Selection
}

\author{
John Sonchack ${ }^{\alpha}$, Adam J. Aviv ${ }^{\beta}$, and Jonathan M. Smith ${ }^{\alpha}$ \\ ${ }^{\alpha}$ University of Pennsylvania, ${ }^{\beta}$ United States Naval Academy
}

\begin{abstract}
Managing an intrusion detection system (IDS) requires careful consideration of the IDS rule set used to match malicious traffic. Network operators face a tradeoff when selecting rules: a rule set that is too conservative (too few rules) could lead to network intrusion and attacks from unforeseen risks, while a rule set that is too broad (too many rules) runs the risk of increasing false alerts and diminishing network throughput. The ultimate goal is to deploy rules that are conservative but proactive, and optimizing and testing such a rule set can be time consuming and limited when considering only locally observed network traffic. We argue that automated techniques to compare feedback from multiple collaborating sources, such as collaborative filtering between networks, can improve local rule sets. Our system, ROCK (Rule set Optimization via Collaborative Knowledge), recommends network-specific, locally untested rules to network operators based on correlations between their feedback and previously submitted feedback from other operators. We evaluated ROCK in two experimental deployments to detect shellcode and in simulation to measure the effect of broad collaboration. We find that network operators begin to see benefits when providing feedback ratings with as few as 5 rules and deploying only the top 5 recommended rules for that network; shellcode detection rates increase by up to $150 \%$ over a local baseline with little to no impact on false alerts. Our simulation analysis suggests that ROCK's recommendation quality increases rapidly with the number of user networks and can leverage varied degrees of similarity across networks. Our results demonstrate how security through collaboration can benefit local networks and provide proactive security in an automated way.
\end{abstract}

Keywords: Network Security, Intrusion Detection, Security Collaboration, IDS, Rule Set 


\section{Introduction}

Intrusion Detection Systems (IDSes) rely on an ever increasing number of rules to detect malicious traffic. Selecting the right rules to load onto an IDS deployment can be a challenging task for network operators. Rules are designed to detect specific threats and may not be equally effective for all networks. If network operators select too broadly, they will detect many threats, possibly even some previously unobserved on the local network, but they also run the risk of deploying rules that are poorly generated or ill-suited to the network services, increasing false alerts and decreasing network throughput. Alternatively, if network operators select a rule set that is too conservative, false alerts will be low but detection rates will also be low. Network operators run the risk of missing previously unforeseen threats leaving network services vulnerable. The goal is to deploy an IDS rule set that is both conservative and proactive, and be able to adapt and update the rule set as information and threats change.

The challenge of meeting this goal is related to determining the effectiveness of IDS rules for a given network. One way to determine effectiveness is by testing rules against samples of local traffic. Unfortunately, this can be time consuming and yield little or no information about rules that do not match any of the sample traffic. Another approach is based on shared information about rule performance from other network operators which can bootstrap the testing and rule selection process.

Collaboration is an increasingly supported network security technique. For example, the United States has recently proposed an initiative [24] to make it easier for private organizations to share threat information, and the European Union is working on a cyber security directive [1] to ensure that organizations across europe share security related information.

In practice, many IDS operators are willing to share information. The Emerging Threats repositority [3] contains an Open Source rule repository consisting only of user submitted IDS rules. It is updated daily and has with over 200, 000 active users. Unfortunately, using shared information to guide the rule selection process is difficult, and requires each IDS operator to manually analyze the shared information and determine how it relates to their network.

Motivated by this challenge, we wish to develop an automated technique to analyze shared information about IDS rule effectiveness, so that IDS operators can more easily collaborate to select the best IDS rules to deploy on their networks. We argue that collaborative filtering techniques, which make recommendations based on small amounts of feedback from multiple collaborating sources, can be applied across networks to recommend IDS rules to network operators. We propose a system called ROCK (Rule set Optimization via Collaborative Knowledge) that is de- 
signed to recommend network-specific, locally untested rules to network operators based on correlations between local feedback and previously submitted feedback from other network operators, much like how Netflix can recommend movies to users based on feedback ratings from similar users that share preferences in the past. In the same way in ROCK, network operators submit ratings of IDS rules, i.e. measurements of rule classification performance on locally collected traffic samples, and ROCK correlates across network based on feedback to predict how rules currently unused at that network would be rated. Essentially, ROCK provides each network operator with customized and personal rule recommendations that can be used to add (or remove) rules from the current rule set.

To evaluate ROCK, we measured its performance in two experimental deployments using data collected from network-diverse honeypot deployments. Using the Snort IDS system [31], two sets of possible rules were considered: rules from the Sourcefire and Emerging Threats repositories, as well as automatically generated rules using Nebula [39]. Because traffic at honeypots are malicious by definition, we instead focused the IDSes on detecting a subset of malicious traffic that contained shellcode as verified by LibEmu [9]. In both deployments, the distributing of shellcode traffic was temporally and geographically diverse, which well models how threats spread on the broader Internet.

We find that ROCK can provide a large boost in detection accuracy with limited affect on false alerts. IDSes that added just the top 5 recommend rules for that network saw an increase in shellcode detection of up to $150 \%$, with little to no impact on the false alert rate. The resulting detection rates were on-par with the maximum achievable using all the rules available, $\sim 20,000$. Surprisingly, network operators did not need to provide a vast amount of feedback on rules to start to see the benefits of collaboration — rating just 5 rules was often sufficient. Providing feedback to ROCK potentially reveals information about internal operations on the network, and the fact that even limited rule feedback in ROCK provides benefits means that operators can tune their participation to maximize the recommendations and minimize the exposure.

Finally, we wish to better understand how ROCK would perform as it scales to more collaborating networks. We implemented a statistical simulation of ROCK with hundreds of collaborating networks sharing feedback on diverse rule sets. We modeled the networks and rule sets based on the live deployments with subsets of networks and rule sets corresponding better than others. We found that the quality of ROCK recommendations increased quickly with the number of users, and that ROCK effectively leverages scale as more networks collaborate.

These result suggest that automated collaboration can greatly benefit security and provide proactive protections as network threats change. To summarize, the papers contributions: 
- Developed ROCK, an automated collaboration engine for recommending IDS rules through feedback from diverse networks;

- Evaluated ROCK using live deployments to recommend rules for shellcode detection;

- Verified ROCK's effectiveness in live deployments by reproducing the results in two different experiments separated in time and network diversity;

- Simulated ROCK to demonstrate that it scales well and performance increases as the number of collaborating networks increase.

The rest of the paper is organized as follows. First, in Section 2, we discuss related work. Next, in Section 3, we describe ROCK and its collaborative recommendation algorithm in more detail. We evaluate ROCK for recommending shellcode detection rules in Section 4, and then extend this analysis using simulation in Section 5. Finally, we discuss concerns related to large scale deployment in Section 6, and then conclude in Section 7.

\section{Related Work}

Network based IDSes, such as Snort [31] and Bro [25], monitor network traffic for predefined suspicious activity or patterns. These systems form an important layer of defense against Internet threats, and are widely deployed. To detect threats, IDSes compare traffic against rules that describe flow level features indicative of maliciousness. Typically, a rule consists of a list of values that must be present in a packet's header fields (e.g., source/destination information), along with a byte sequence that must also be present or a regular expression that match the flow's contents (e.g., a signature). When a flow matches one or more rules, the IDS generates an alert that is later reviewed by the IDS operator. The effectiveness of an IDS at classifying traffic depends on the rules that it uses. The goal of ROCK is to recommend rules to IDS deployments that will increase their effectiveness.

Several systems have been developed that automatically generate rules $[27,17$, $23,22,41,8,38,39]$. These systems mostly operate by finding expressions or byte sequences that are shared by many malicious traffic flows. Automatic rule generators, in theory, allow rule based network defense to remain effective against rapidly evolving and diverse threats. While some techniques such as Argos [27] refine their rules, these systems do not in general address the problem of determining which IDS deployments should use generated rules, which is increasingly important as the numbers of threats, vulnerabilities, rules, and rule generation techniques grow.

To our knowledge, ROCK is the first system to address this problem by providing IDS operators with recommendations about which rules to add to their rule 
sets. Current best practices for rule selection rely on manual analysis [35] and knowledge sharing via mailing lists and rule repositories [5, 3]. ROCK automates this collaborative knowledge building and rule selection process by applying the machine learning technique of collaborative filtering [7, 36]. These algorithms have been successful in systems recommending products [19], movies [16], news articles [30], and emails [14].

Although ROCK is, to our knowledge, the first application of collaborative filtering to IDS optimization, it is not the first application of collaboration to the network security. Blacklist generation systems such as [42] and [43] leverage collaboration for proactive defense. These systems have different goals than our work; they provide networks with IP address blacklists, whereas we provide considerably more general IDS rule recommendations. Furthermore, the methods used in their systems are not immediately applicable to the domain of IDS defense.

Other network defense systems leverage collaboration, but at different and complementary scales. Alert Fusion systems [10, 32] correlate alerts generated by multiple components of a single network's defense to improve alert quality. Gnort [37] applies collaborative strategies at the processor level, using multiple collaborating Graphics Processing Unit (GPU) cores to match a known set of IDS rules. Collaboration amongst classifiers in learning systems has also been proposed [13], with a primary focus of robust pattern classification (including detection of novel attacks [18]). Yegneswaran, et al. [40] designed and implemented a large scale overlay system to detect global attack trends. These techniques are all complementary to ROCK, which builds knowledge about rules to make recommendations optimized for specific IDS deployments.

ROCK is also related but orthogonal to the area of IDS testing. Systems such as [33] focus on generating traffic to measure and compare the effectiveness of IDSes viewed as black boxes. ROCK, on the other hand, correlates measurements on real traffic to predict the effectiveness of IDS rules, in order to optimize the effectiveness of any rule based IDS at a given network.

\section{System Overview}

ROCK, depicted in Figure 1, recommends IDS rules to network operators. An active ROCK user submits rule ratings, or measurements of rule classification performance on locally collected traffic samples. ROCK quantifies the similarity between the active user's ratings and the ratings provided by other network operators, and predicts which rules would be most effective at the active user's network based on how other similar network operators rated the rules.

ROCK is designed to overcome two main challenges that network operators face in selecting rules: 


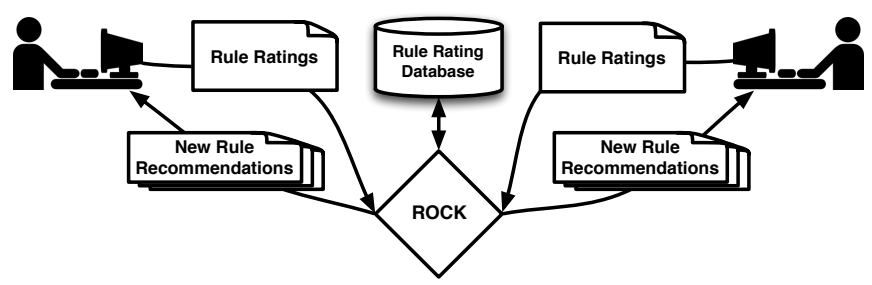

Figure 1: ROCK correlates feedback about rule effectiveness from many network operators to provide specific rule recommendations for each network operator.

- Rule effectiveness varies, and is network dependent: there are many rules, written for specific threats and scenarios, and these conditions are not ubiquitous across networks.

- Rule effectiveness is difficult to measure locally: testing rules against local traffic is time consuming, and even if a rule is important for a network's defense, it may not match traffic in a previous trace of traffic from that network.

We observed these challenges directly in our experimental data, and discuss them further in Section 4.3. ROCK overcomes the limitations of local analysis by gleaning ratings from the submissions of many users, and deals with the nonuniform nature of rule effectiveness by analyzing correlations between users to make specific recommendations for each user.

ROCK's collaboration scheme assumes that all of the collaborators have access to the same rules, and that each collaborator wants to select which subset of the rules are best to load onto their IDS deployment, while performing a minimal amount of work themselves. However, ROCK, and collaborative filtering algorithms in general, only require a small number of ratings from each user. Therefore, in scenarios where different collaborators have access to different subsets of rules (e.g. different collaborators use different rule repositories), we hypothesis that ROCK will still be able to make effective recommendations, as long as there is some overlap between pairs of operators with respect to what rules they rate. Our experiments in Section 4, support this hypothesis.

In the remainder of this section, we discuss the machine learning technique that ROCK implements, collaborative filtering, and then the ROCK algorithm itself.

\subsection{Collaborative Filtering}

Collaborative filtering algorithms are a class of machine learning algorithms designed to predict the relationship between users and items; specifically, they are designed to predict how users will preferentially score items that they have not yet 
encountered. There are several mathematical interpretations of this problem, each of which has a separate class of solutions, as surveyed by $\mathrm{Su}$ [36]. One of the most intuitive interpretation is given by user-based collaborative filtering algorithms. These algorithms are based on the assumption that if two users have scored some items similarly, then they have similar behaviors or preferences, and will likely rate other items similarly. Thus, to predict how user $X$ will rate item $n$, a user-based collaborative filtering algorithm analyzes how other users with rating patterns similar to $X$ have rated item $n$. These algorithms are not sensitive to content, and have been applied to a number of domains, as we discuss in Section 2.

This user-based approach to collaborative filtering maps well to the problem of collaboratively building knowledge about where IDS rules are effective, and recommending them to network operators: two users who rate rules similarly are likely to be defending similar network services; in turn the ratings of one user should hold greater than average weight for predictions about the other user, and vice versa.

\subsection{The ROCK Algorithm}

Specifically, given $U$ users, $R$ rules, and a common rule effectiveness metric $M$, ROCK functions as follows:

1. Each user $j$ measures the effectiveness of some subset $R_{j}$ of the rules in $R$ using $M$, and submits these scores to ROCK, along with $N_{j}$, the number of recommendations user $j$ requests from ROCK.

2. ROCK computes the similarity between each pair of users $j$ and $k, S(j, k)$, by comparing $R_{j}$ and $R_{k}$. If there is no overlap between the rules rated by $j$ and $k$, ROCK sets $S(j, k)=0$. Otherwise, ROCK converts $R_{j}$ and $R_{k}$ into vectors of length $|R|$, where unrated rules are temporarily assigned a rating of $0 . S(j, k)$ is then computed by measuring the difference between the two vectors with the Quadratic-Chi Histogram Distance Metric [26]. Experimental results show that this metric provides a more accurate estimate of the similarity between vectors than other popular methods such as [21] and [20].

3. ROCK computes a predicted rule score vector $\hat{\vec{S}}_{j}$ for each user, which contains a predicted score for all rules measured at any network, using Equation 1, which states that the predicted effectiveness score of a rule for a specific user is the weighted average of all scores submitted for the rule by other users, where the weight of score is the similarity between each scorer $k$ and the current user requesting the score $j$.

4. Finally, for each user $j$, ROCK ranks the rules by their predicted scores (i.e. by $\hat{\vec{R}}$ ), and returns the top $N_{j}$ highest rated rules to user $j$. 


$$
\hat{\vec{S}}_{j}[r]=\sum_{k \in U \text { sers }} R \vec{S}_{k}[r] * S(j, k)
$$

\subsection{Complexity Analysis}

Time Complexity. The worst case time complexity of computing a set of recommendations for a single user is $O(U * R+R \log R)$, where $U$ is the number of users and $R$ is the number of rules. More specifically, we can break this complexity down into three components:

- Similarity Computation The Quadratic Chi similarity metric computes the similarity score for a pair of vectors in linear time, with respect to the length of the vectors. In this case, each vector is a user's rule ratings (at most $R$ ratings). To compute recommendations for a single user, ROCK could, in the worst case, need to recompute the similarity between the user and all other users (at most $U$ users). Thus, the complexity for similarity computation is $(U * R)$.

- Score prediction Score prediction for a single user is a multiplication of two vectors: the similarity vector that measures how similar the user is to each other user (size $U$ ); and the rules for which ratings need to be predicted (at most size $R$ ). Thus, the complexity for score prediction is also $(U * R)$.

- Rule Ranking Sorting the $R$ rules by their predicted scores can be done in $R \log R$.

We discuss the performance of an un-optimized C++ implementation of ROCK in Section 6.

Space Complexity. The space complexity of ROCK is dominated by the ratings matrix, which is size $O(U * R)$, and the user correlation matrix, which is size $O\left(U^{2}\right)$. In practice, the ratings matrix is likely to be sparse, since each user is only likely to provide ratings for a small number of rules. Thus it could be stored in a sparse matrix data structure, which would provide a space complexity equal to the number of non-zero ratings that have been provided by users. To reduce space complexity, each row of the correlation matrix can be computed as it is needed (i.e. instead of storing the entire correlation matrix, wait until user $\mathrm{X}$ makes a request for new recommendations and then compute the correlation between $\mathrm{X}$ and all other users). 


\section{Live Deployment Evaluation}

In this section, we evaluate ROCK in a live deployment scenario. Our main goal is to compare rule sets built with ROCK recommendations to rule sets built using the current standard approach, in which each IDS operator analyzes rules autonomously and then selects rules based only on their own analysis.

More specifically, we evaluate ROCK's ability to recommend rules for Snort [31] IDSes monitoring honeypot traffic for shellcode. We test ROCK using this setup for two reasons. First, it demonstrates a practical use case for ROCK; honeypots are widely deployed and considered valuable tools for production networks and capturing malicious binaries. Optimizing the effectiveness of the IDS systems that monitor such honeypots, and reducing the amount of configuration and maintenance work required, would directly benefit many network operators.

Second, evaluating ROCK with real rules requires traffic traces from multiple networks that contain content. In general, such data is difficult to acquire, analyze (i.e. to find ground truth in), and share; limiting the reproducibility and scope of experiments [34]. We chose an experimental setup overcomes many of these issues. Honeypot software is publicly available (Dionaea [15]) and can be easily deployed in diverse network settings to collect data. The experiments also make use of publicly available IDS rules (Sourcefire [5] and Emerging Threats repository [3]) and open source automatic rule generators (Nebula [39]), and ground truth is determined using a publicly available shellcode detector (LibeEmu [9]). In fact, we were able to reproduce results in two deployments of ROCK using the same experimental setup.

Further, by focusing on detecting shellcode, which constitutes less than half of the traffic observed at our honeypots, as Table 1 summarizes, we can use the honeypot traffic to not only evaluate how ROCK recommended rules affect the detection rate of IDSes, but also the false alert rate of IDSes. Additionally, the detection rates could be seen as lower bounds to the actually detection rate if all IDS rules were considered, including those that detect a wider range of malicious traffic.

In the remainder of this section, we describe our experimental set up in more detail, and then discuss experiments that analyze ROCK's effectiveness and benefits as a rule recommender in this scenario.

\subsection{Experimental Setup}

Data. We collected two sets of traffic for the live deployment experiments. In initial analysis, we used traces from 5 honeypot servers collected from February 14-24, 2011, and we were able to reproduce the results in a following live deployment from 6 servers using data collected from March 21-31, 2014. In both 


\begin{tabular}{|l|ccccccc|}
\hline Day & 1 & 2 & 3 & 4 & 5 & 6 & 7 \\
\hline & \multicolumn{7}{|c|}{ February, 2011 Data Set } \\
Unique IP Sources & 275 & 486 & 574 & 602 & 601 & 615 & 649 \\
Flows & 7914 & 25519 & 29682 & 89974 & 122131 & 150254 & 157805 \\
Shellcode Flows & 1786 & 12107 & 9937 & 37545 & 58403 & 69035 & 74367 \\
Repository Matches & 4872 & 11790 & 15839 & 45129 & 56727 & 67901 & 71328 \\
Nebula Matches & 2634 & 10760 & 10040 & 43257 & 55335 & 66454 & 69161 \\
\hline & \multicolumn{7}{c}{ March, 2014 Data Set } \\
Unique IP Sources & 446 & 446 & 468 & 561 & 538 & 519 & 449 \\
Flows & 40144 & 54827 & 38664 & 50962 & 71863 & 89478 & 39587 \\
Shellcode Flows & 19308 & 26396 & 18230 & 24513 & 34484 & 43393 & 18856 \\
Repository Matches & 14597 & 20237 & 14148 & 18678 & 26220 & 32301 & 11269 \\
Nebula Matches & 14641 & 19964 & 13811 & 18559 & 26101 & 31933 & 11236 \\
\hline
\end{tabular}

Table 1: Summary of traffic used to test ROCK in Section 4.

cases, we used the first 3 days of traffic to generate rules using Nebula [39], and the remaining 7 days to test ROCK's ability to recommend these rules. The IDS rules used in recommendation were all design for the Snort IDS and were either culled from the Sourcefire or Emerging Threats snort rule repository or automatically generated using Nebula. Table 1 summarizes the traffic from the testing days of our of data sets. In all the experiments, we used these traces to model a scenario where the services hosted by each honeypot server were monitored by a separate Snort [31] IDS deployment and managed by a separate network operator.

Data Collection Servers. The honeypot servers in the experiments ran Dionaea [15], which provides emulated HTTP, FTP, MSSQL, MySQL, SMB, and SIP services. In both experimental periods, the servers were diversely located, both geographically and IP-space diverse. Despite offering the same basic services, there were several important differences in the traffic collected.

In the 2011 data set, two of the servers had IP addresses in the random scanning range of Conficker [12], and a large proportion of the traffic they collected were exploit attempts from that worm. Also, in 2011, one of the servers was hosted on a home DSL line, and changed IP addresses several times through the data collection, causing it to be targeted by different types of malware. Finally, two of the servers were hosted at data centers, and received significantly more MSSQL attack traffic than other servers.

In the 2014 data set, the distribution of servers were similarly diverse in geographic and network space; however, their exposure to malicious traffic was less diverse. One reason for this could be because all servers were hosted in data centers as opposed to home and institutional networks. Another significant difference is the Conficker worm has greatly diminished [4], which limited the number of shellcode exploit attempts observed. Despite the decrease in attacks, we found that 


\begin{tabular}{|c|c|c|}
\hline Rule Class & Class Summary & $\begin{array}{l}\text { Class Size } \\
\text { (\# of Rules) }\end{array}$ \\
\hline Web Application Attack & Detect application layer exploits (e.g. buffer overflows.) & 6101 \\
\hline Attempted User Exploit & & 4446 \\
\hline Attempted Admin Exploit & & 1386 \\
\hline Trojan Activity & $\begin{array}{l}\text { Detect application and network layer activity indicating } \\
\text { that local hosts are infected. (e.g. connection attempts } \\
\text { to IP addresses or domains known for distributing malware.) }\end{array}$ & 5688 \\
\hline Policy Violation & $\begin{array}{l}\text { Detect application layer policy violations. (e.g. outbound file } \\
\text { transfers over chat applications.) }\end{array}$ & 1221 \\
\hline Attempted Recon & $\begin{array}{l}\text { Detect network layer information gathering (e.g. traceroute } \\
\text { and netmap), and attempts to access general network resources } \\
\text { such as NFS mounts. }\end{array}$ & 695 \\
\hline
\end{tabular}

Table 2: Some classes of rules from the Sourcefire and Emerging Threats repositories that matched traffic in our live deployment data sets.

ROCK was still effective at identifying and recommend IDS rules that would be effective at each network.

Due to space limitations and greater familiarity, our discussion focuses on experiments from the 2011 data set unless there were significant differences between results from the two data sets.

Intrusion Detection Systems. In these experiments, we used ROCK to tune the rule sets of Snort [31] deployments. We chose Snort over other IDSes such as Bro [25] and Suricata [6] because it is currently the most widely used Open Source IDS, with a large community of users that already collaborate and share information (e.g. by sharing rules in repositories such as Emerging Threats [3]).

The Snort rules that we evaluated are based on regular expressions, and generate alerts whenever they match a packet or TCP / UDP stream. Regular expression based detection is an important part of many IDSes, including Bro and Suricata, and so our results in this section are directly relevant to using ROCK with those other systems. Further, ROCK is designed to be agnostic to IDS platform, and our hypothesis is that it would be applicable to any rule based IDS. It treats each rule as an abstract item, and recommen- dations are only based on rule effectiveness ratings. The statistical simulation in Section 5 analyzes ROCK independent of IDS platform, and supports the hypothesis that ROCK would generalize to other IDSes.

Rule Sources. To study ROCK's performance in a realistic setting, the experiments in this section use rules from well known, publicly accessible Snort repositories, and a recently proposed Snort rule generation system, summarized below:

1. Snort Repositories: approximately 20,000 rules obtained from the popular Sourcefire [5] and Emerging Threats (E.T.) [3] repositories. Since new rules are added frequently, we only used rules that were added before the 
data collection dates, for all experiments. The rules from these repositories are diverse, and match many different traffic features at both the network and application layers. Table 2 describes several classes of rules from these repositories that matches traffic in our experiments. ${ }^{1}$

2. Nebula: approximately 650 rules generated by a Nebula system, on traffic collected at our servers in the three days preceding ROCK testing. Nebula clusters traffic flows to generate rules, and has two main parameters: $C$, the cluster density; and $N$, the number of flows to add to a cluster before attempting to generate a rule. We selected parameters of $C=95$ and $N=5$ because we found that they produced the best trade off between maximizing true positive rate and minimizing false positive rate in cross validation trials over the data used to generate rules.

In the remainder of these experiments, we call the set of all rules from each of these sources Snort Rules and Nebula Rules, respectively. ${ }^{2}$

The quality of the provided rules are not uniform. Many of the rules do not provide high detection rates and low false positive rates. For example, using all of the rules described above, only about $60 \%$ of the shellcode containing flows are detected in the 2011 data set with a high false alert rate $>25 \%$, which further demonstrates the challenges faced by IDS operators. We found that the primary reason for the low detection rates was polymorphic shellcode, which many current regular expression based rules have difficulty detecting. In these experiments, we only consider shellcode exploit attacks so that we can obtain an objective ground truth. In practice, detection rates would likely be higher because IDS operators are interested in many other classes of threats where polymorphic shellcode cannot be used (e.g. port scans and injection attacks).

While the effectiveness of these rules against shellcode is dissapointing, it is important to note that it is not the goal of ROCK to generate better rules for detecting shellcode, which is a highly motivated and challenging problem but outside the scope of this research. Rather, the goal of ROCK is to help network operators find the available rules that will be the most effective on their networks. The fact that so many of the available rules have issues with respect to detection and false alert rates further motivates the need for a rule recommendation system like ROCK.

Rule Effectiveness Metrics. ROCK requires users to rate the effectiveness of a subset of the available rules on their IDS deployments. In these experiments, we

\footnotetext{
${ }^{1}$ Rule class is based on metadata provided by the repositories.

${ }^{2}$ We note that Nebula rules are also technically "Snort rules", however we use these labels for more concise discussion.
} 
assume that a rule rating is an estimate of its accuracy as a classifier that labels traffic flows as either malicious or non-malicious. We define accuracy as the percentage of tested flows that the rule labeled correctly. We use accuracy because it is a metric designed to take many of the possible kinds of classification errors that an IDS operator might care about into account. Further, in preliminary experiments, we found that rating rules based on accuracy led to better ROCK recommendations than other binary classification metrics such as sensitivity, specificity, or precision. Although user submitted ratings are estimates of accuracy, we evaluate ROCK recommendations based on how they affect IDS effectiveness using multiple metrics.

Evaluating IDSes and rules with these metrics requires an estimate of ground truth, or the knowledge of which traffic is truly malicious. While it is challenging to know the ground truth exactly, we estimated it at the per-flow level based on shellcode detection via the LibEmu [9] shellcode emulator.

Experimental Trials. For both data sets, we divided the 7 days of testing data from all servers into 2 hour trials. For each trial, we used data from the 1 to 12 hours preceding it as a training window. In the training, at each server, we scored rules based on the server's training data and submitted the scores to ROCK to receive new recommendations. All newly recommended rules during the trial period represent proactive defenses, and the detection rate of those rules represent an increase in performance of the IDS as a whole. Since the training window varies, we specify our training windows in all experiments.

Baseline Rule Sets. In several of our experiments, we compare ROCK recommended rules with rules selected using a baseline heuristic based entirely on local information. The baseline rule set, for a server $S$ in a trial $T$, is a rule set selected using only the traffic locally observed by $S$ during the training period preceding $T$. Baseline rule sets allow us to quantify the benefit of IDS operators collaborating and using ROCK recommendations to build rule sets instead of acting autonomously. Specifically, the baseline rule set for server $S$ in trial $T$ is generated as follows:

1. Score the accuracy of all rules during the training period preceding $T$.

2. Rank the $N$ rules that matched traffic during this period based on their accuracy.

3. Select the top $\frac{N}{2}$ rules from the ranked list.

Our choice of the top $\frac{N}{2}$ rules is motivated by the observation that in practice, network operators optimize for both high true positive rates (or detection) and low false positive rates (to minimize false alerts and increase IDS usability). We found that on average, across all the experimental trials using both rule sources, this fraction provided the highest ratio of true positives to false positives. 


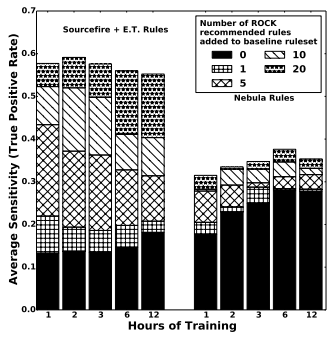

Figure 2: Average IDS detection when ROCK recommended rules are added to locally selected baseline rule sets in the 2011 data set.

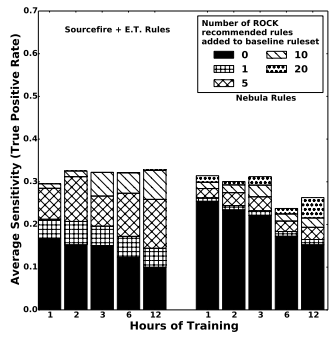

Figure 3: Average IDS detection when ROCK recommended rules are added to locally selected baseline rule sets in the 2014 data set.

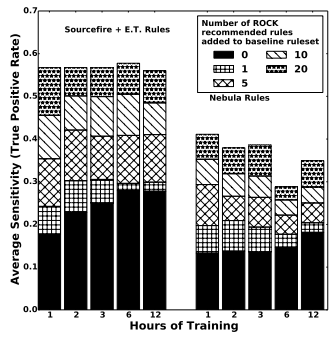

Figure 4: Average IDS detection when ratings of rules from one source were used with ROCK to obtain recommendations of rules from a different source.

Generalizing Results. Our scenario allows us to evaluate a practical use of ROCK in a controlled environment. We also make the argument that ROCK's effectiveness in this scenario is a lower bound on its effectiveness in other scenarios:

- Since shellcode is one of many types of malicious traffic, such as port scans and injection attacks, our results provide a lower bound on the increased detection an IDS operator can achieve by adding ROCK recommended rules.

- Since the rules we use are good enough to be included in a well maintained public repository, or generated by a published rule generation algorithm, they should be less likely to produce alerts on legitimate flows than on unwanted or malicious flows. Our honeypots do not offer any actual services, whereas real networks do, so the ratio of legitimate to unwanted or malicious traffic should be significantly higher on real networks. Thus, our results provide an upper bound on the increased false alert rate an IDS operator would observe when adding ROCK recommended rules.

\subsection{Detection Rates}

Using both our 2011 and 2014 data sets, we evaluated how adding ROCK recommended rules to locally selected baseline rule sets affected the true positive rate, or percentage of malicious flows detected, of the IDS deployments. Figures 2 and 3 show the true positive rates of the baseline rule sets, and the baseline rule sets with different numbers of ROCK recommended rules added to them. Each bar shows the average sensitivity, across all trials and IDS deployments; the bars on the left of each figure depict experiments where the baseline and ROCK recommended rule 


\begin{tabular}{|ll|rccc|}
\hline & Num. Rec. Rules & 1 & 5 & 10 & 20 \\
\hline \multirow{2}{*}{2011} & Snort Rules & $38.68 \%$ & $152.37 \%$ & $228.65 \%$ & $295.44 \%$ \\
& Nebula Rules & $12.26 \%$ & $104.55 \%$ & $165.85 \%$ & $219.25 \%$ \\
\hline \multirow{2}{*}{2014} & Snort Rules & $20.29 \%$ & $76.21 \%$ & $76.45 \%$ & $76.45 \%$ \\
& Nebula Rules & $12.86 \%$ & $63.79 \%$ & $63.99 \%$ & $63.99 \%$ \\
\hline
\end{tabular}

Table 3: Detection rate percentage increases, averaged over all training windows, for data sets, rule source, and recommendation counts.

sets were composed only of Snort rules, and the bars on the right of each figure depict experiments where the rule sets are composed only of Nebula rules. In all cases, adding ROCK recommended rules significantly increased the average detection rate. As previously discussed, we believe that the detection benefit of ROCK would be even greater in scenarios where network operators were seeking to detect other classes of malicious traffic in addition to shellcode.

These results also demonstrate how ROCK is limited by the detection rates of the available rules. The Snort Rules were much more sensitive to malicious traffic than the Nebula Rules, and when the IDS deployments used these rules with ROCK, they achieved higher detection rates. More importantly, comparing the data from 2011 to the data from 2014 reveals that both rule sets detected a significantly smaller percentage of the malicious traffic. We used up to date rules, relative to when the data sets were collected, but were only able to achieve an approximate $30 \%$ detection rate for shellcode in the 2014 data set using all the over 20,000 rules from both Snort repositories and Nebula. Table 3 summarizes the detection rate increases for both data sets.

This decrease in detection rate correlates with two trends. First, the diminishing of Conficker [12]: Microsoft found that the number of hosts reporting Conficker infections decreased by almost $30 \%$ in 2012, and 50\% in 2013 [4]. Snort was effective at detecting Conficker because it was a well known threat that exploited a small number of vulnerabilities, and thus both repositories and Nebula could easily provide rules to detect it. The second trend that may have affected Snort's detection rate is the general advancement of Internet threats, which exploit an ever more diverse array of vulnerabilities with increasingly advanced techniques. Despite the decrease in performance of Snort, ROCK was still able to pick out the few rules that were most effective at detecting shellcode at each IDS deployment and recommend them first, demonstrating its robustness.

Rule generation systems are one proposed method for dealing with the increasingly advanced techniques of modern malware [23, 28]. However, these systems require significant operator knowledge to deploy and maintain, limiting their deployment. Using our experimental data, we explored whether ROCK allowed network operators without the resources to deploys these systems to still gain the 


\begin{tabular}{|c|cccc|}
\hline Rule Group & \# IDSes & \# Rules & Accuracy Average & $\begin{array}{c}\text { Accuracy Std. Dev. } \\
\text { (Across IDSes) }\end{array}$ \\
\hline 1 & 5 & 14 & 0.718 & 0.186 \\
2 & 4 & 15 & 0.612 & 0.141 \\
3 & 4 & 3 & 0.659 & 0.153 \\
4 & 4 & 8 & 0.727 & 0.212 \\
5 & 3 & 1 & 0.678 & 0.224 \\
6 & 3 & 2 & 0.743 & 0.195 \\
7 & 3 & 10 & 0.642 & 0.173 \\
8 & 3 & 7 & 0.79 & 0.194 \\
9 & 3 & 1 & 0.703 & 0.15 \\
10 & 2 & 38 & 0.519 & 0.005 \\
11 & 2 & 6 & 0.796 & 0.091 \\
12 & 2 & 1 & 0.755 & 0.235 \\
13 & 2 & 3 & 0.941 & 0.054 \\
14 & 2 & 7 & 0.703 & 0.184 \\
\hline
\end{tabular}

Table 4: Statistics about groups of rules that matched traffic at different unique subsets of IDSes in our experimental data.

benefits of their rules. Specifically, we performed trials using our 2011 data set where at one IDS, which we will refer to as the testing IDS $S$, we used the training data to score only rules from one rule source. At all of the other IDSes, during that training period, we scored rules from both sources. We submitted all of these scores to ROCK, and then evaluated the detection rate of rule sets at $S$ that included the baseline rule set plus ROCK recommended rules that were from the source $S$ did not score in training.

Figure 4 shows the results of our experiment, averaged across trials in which each server acted as the training server, and then averaged across training servers. On the left of this figure are trials where the training servers used Nebula Rules to train, and added ROCK recommended rules that came from the Snort Rule Set, while on the right are trials where the training servers used Snort Rules to train, and added ROCK recommended rules that were generated by Nebula. Comparing these results to those depicted in Figure 2 demonstrates that ROCK can effectively recommend rules from a repository or rule generator, even to users who have never rated rules from that source, overcoming a barrier to large scale usage of automatically generated rules. As users of ROCK deploy novel rule generation techniques, other collaborating users automatically become informed about new rules, without dedicating any additional effort or changing their practices.

\subsection{Deployment Specific Recommendations}

ROCK analyzes the relationship between rules and IDSes. To understand ROCK better, we examined the relationship between rules and IDS deployments more closely. More specifically, we grouped all the rules from both the Snort and Nebula Sets into non-overlapping groups, based on the subset of IDSes where 


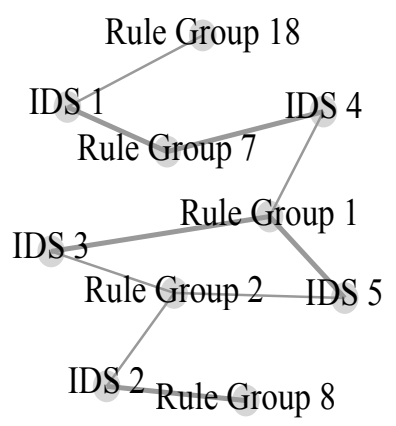

Figure 5: Graph of the relationship between IDSes and select rule groups. An edge between an IDS deployment node and a rule group node exists if the rule group was one of the top 2 most accurate for the IDS.

they matched traffic. Table 4 summarizes the 13 non-overlapping sets of rules that matched traffic at two or more of our IDS deployments. We found that accuracy (i.e. the fraction of flows the group of rules classified correctly, averaged across all IDSes) varied greatly across rule groups, ranging from .61 to .94, and demonstrating the need for careful rule selection. More importantly, as the last column of Table 4 shows, the accuracy of a single rule group varied significantly from network to network. This variation makes it difficult for network operators to gain the benefits of collaboration that we highlight throughout the paper.

Although absolute rule effectiveness varied greatly across rules and networks, we also found correlations amongst networks with respect to relative rule effectiveness. Figure 5 shows these correlations, with a graph containing edges between IDS deployments and the top two most accurate rule groups for each deployment. $\frac{3}{5}$ of the rule groups that were highly effective at one IDS (based on the top two metric) were highly effective at other IDSes. Further, at least one of the two most effective rule groups at every IDS was also highly effective at another IDS.

ROCK is designed to quantify and leverage these correlations to make customized recommendations to each collaborating network operator. Using trials with 2 hour training periods followed by 2 hour testing periods, we tested whether ROCK recommended different rules to different IDS deployments by measuring the overlap between sets of ROCK recommended rules to different pairs of IDSes, as the number of recommended rules varied. We measured the overlap between the recommendations that ROCK provided to IDSes, $p$ and $q$ as the number of rules ROCK recommends to both $p$ and $q$ divided by the number of rules ROCK recommends to a single IDS. Figure 6 shows the average overlap, and the range of overlap values we observed, for both Snort and Nebula Rule Sets. Overlap was small when ROCK recommended few rules, and varied greatly depending on the 


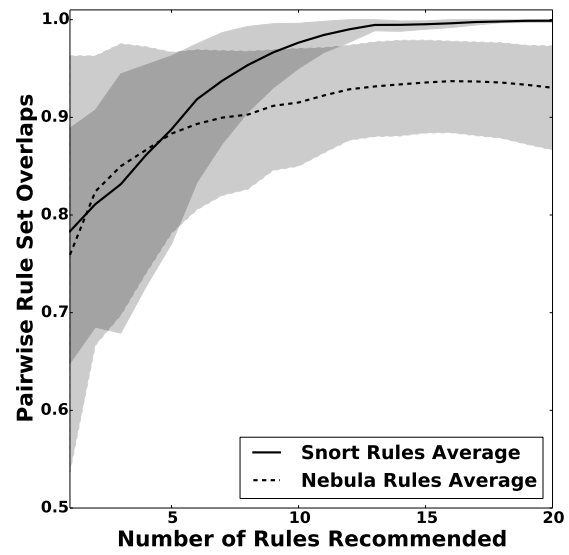

Figure 6: Overlap of the rules ROCK recommended to different pairs of IDSes, as recommendation count varies.

pair of IDSes considered, illustrating that ROCK recommended different rules to different networks. Overlap was generally lower when ROCK recommended Nebula Rules for two main reasons. First, there were fewer Snort rules that matched shellcode traffic as compared to the Nebula rules, which accounts for the slower rate of overlap across networks as more rules are recommended.

Second, the performance of Nebula rules was more network dependent, which caused more variance in the rule ratings provided to ROCK for Nebula rules, and in turn led to greater variation in recommendations. Some rules in the Snort Rule Set also performed differently across our deployments, however there were a small number of Snort rules that were highly effective at most deployments (e.g. Conficker detection rules, as previously discussed). We confirmed that ROCK frequently recommended these rules, contributing to the rapid increase of overlap when recommending Snort Rules, and demonstrating that ROCK is effective at recommending not only rules that are only useful at specific networks, but also rules that are broadly effective across many networks. Overall, these experiments support our hypothesis that ROCK is robust to many factors and would be beneficial to network operators in many scenarios.

\subsection{Rule Effectiveness}

ROCK's goal is to recommend rules to network operators that will effectively classify between malicious and innocuous traffic on their IDS deployments. There are many different ways to measure the effectiveness of such binary classifiers and account for different biases in their operation. In our experimental deployment, we 


\begin{tabular}{|c|ccc|}
\hline $\begin{array}{c}\text { Metric } \\
\text { Recommendations }\end{array}$ & Specificity & Precision & Accuracy \\
\hline 0 (baseline) & 0.798 & 0.381 & 0.6 \\
1 & 0.822 & 0.38 & 0.577 \\
5 & 0.887 & 0.441 & 0.602 \\
10 & 0.899 & 0.441 & 0.612 \\
20 & 0.893 & 0.438 & 0.605 \\
\hline
\end{tabular}

Trials using rules from Snort Set.

\begin{tabular}{|c|ccc|}
\hline $\begin{array}{c}\text { Metric } \\
\text { Recommendations }\end{array}$ & Specificity & Precision & Accuracy \\
\hline 0 (baseline) & 0.771 & 0.452 & 0.585 \\
1 & 0.846 & 0.451 & 0.607 \\
5 & 0.903 & 0.453 & 0.617 \\
10 & 0.919 & 0.453 & 0.618 \\
20 & 0.934 & 0.454 & 0.616 \\
\hline
\end{tabular}

Trials using rules from Nebula Set.

Table 5: Average classification performance for rules in the baseline rule set, and the baseline rule set with added ROCK recommended rules.

measured the classification performance of rules recommended by ROCK using several common binary classifier metrics. Table 5 shows the average specificity (percentage of non malicious flows correctly classified), precision (percentage of alerted flows that were malicious), and accuracy (total percentage of flows correctly classified) of rules in the baseline and ROCK augmented rule sets, averaged over trials with a 2 hour training period. At a high level, these measurements show that ROCK tended to recommend rules that classified at least as well as the rules selected using the local baseline heuristic.

Deeper trends in these measurements also reveal biases of ROCK. The specificity increased while the other metrics did not, indicating that ROCK is biased towards rules that avoid generating false alerts over rules that avoid other classification errors. This bias aligns with a cautious rule selection strategy that network operators frequently take in practice that values IDS usability over detection rates. It also limits the amount of harm ROCK can cause with poor recommendations: modern IDSes can match traffic quickly against a large number of rules, so adding some rules that do not match traffic at a network, but would be accurate if they did, is significantly less harmful than adding inaccurate rules that inundate an IDSes $\log$ with false alerts.

A cautious rule selection strategy is also important in scenarios where there is overlap with respect to what threats a rule detects. For example, if there are two rules that both detect a single threat, ROCK will recommend the rule that it predicts will have the lower false alert rate. Additionally, even if ROCK highly recommends 


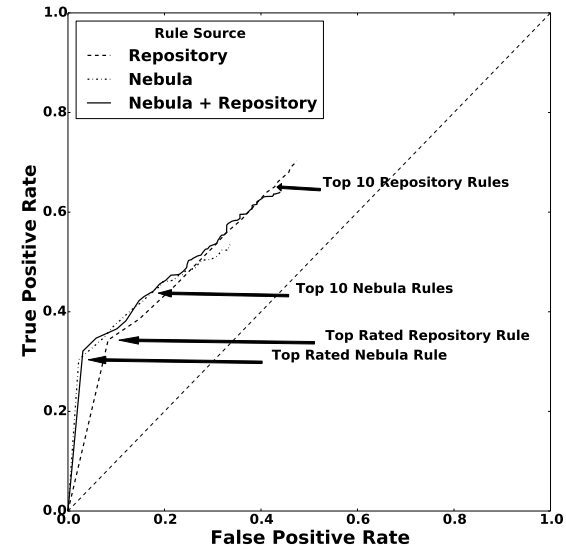

Figure 7: False positive rate vs true positive rate of rules sets consisting only of ROCK recommendations, as recommendation count varies.

a rule that detects a threat that can already be detected with a previously loaded rule, ROCK's bias towards low false alerts mean that the additional rule is not likely to have a large detrimental effect.

We also observed that the average rule precision increased significantly when IDSes used ROCK recommended rules from the Snort Set. This was primarily due to a small number of precise but infrequently matching rules. In many trials, these rules did not show up on many of the baseline rule sets, due to not matching traffic at the respective IDSes during training. However, ROCK was able to recommend the rules to all the IDSes because it analyzed ratings from the few where the rules did match traffic during training. In most cases, we found that we needed to increase the training period from 2 hours to approximately 12 hours for these rules to be included in the baseline rule sets selected using local traffic.

Ideally, ROCK would recommend the rules that were most effective at a network first, before recommending less effective rules. To evaluate the order of ROCK's recommendations, we computed the true and false positive rates of the IDSes in our trial using rule sets consisting of only varying numbers of ROCK recommended rules. Figure 7 is a plot of the (False Positive Rate, True Positive Rate) pairs for between 1 and 20 ROCK recommendations, averaged across all trials, with ratings taken from the 2 hour period proceeding each trial. We repeated this experiment with three rule sources, which are depicted in this figure: only rules from the Nebula Set, only rules from the Snort Set, and a combined rule set containing rules from both sources. For all three rule sets, ROCK recommended the 


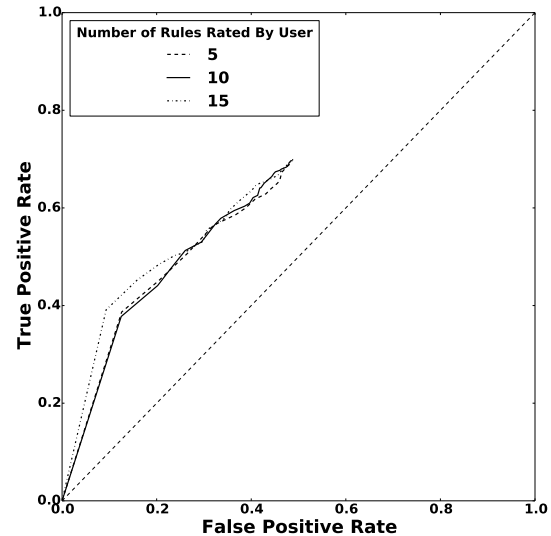

Figure 8: Average False Positive vs True Positive Rate of IDSes as they score different numbers of rules for ROCK and deploy different numbers of ROCK recommended rules.

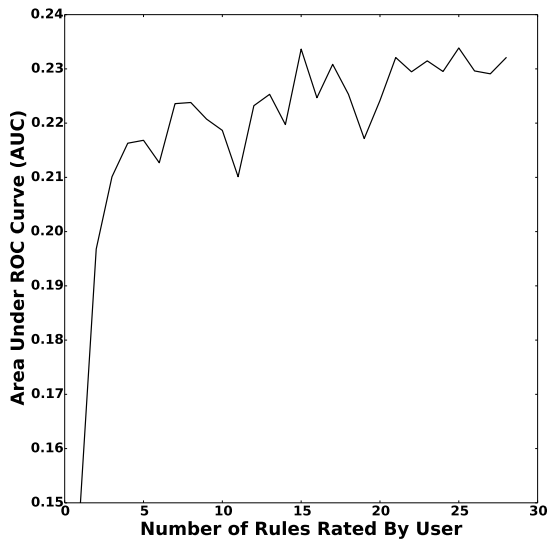

Figure 9: Number of ratings provided by each user vs average area under the ROC curve for number of recommended rules deployed.

rules that provided the most benefits first: on average the first 5 recommended rules detected over $50 \%$ of the shellcode traffic detectable with all of the available rules, but generated less than $10 \%$ as many false alerts. Further, rules that decreased the ratio of true alerts to false alerts were not recommended until there were few better rules left unrecommended.

This experiment also demonstrates that ROCK is limited by the rules that are available: most of the rules in both of these rule sets had high false alert rates for shellcode detection, and so even if rules were selected optimally, it would be impossible to achieve a high detection rate without a high false alert rate. As we have previously argued, this property suggests that our results represent a lower bound on the effectiveness of IDS rules and the benefit of ROCK(i.e. an upper bound on false positive rates and a lower bound on true positive rates).

\subsection{Reducing Operator Work}

With the ever growing number of threats and IDS rules, selecting and maintaining a rule set is time consuming for network operators. Thus, a natural question about ROCK is: how much work is required for a network operator to receive useful recommendations from ROCK? To answer this, we measured the effectiveness of ROCK recommendations as the number of rule scores submitted from each IDS deployment varied. Specifically, for each trial we rated between 1 and 25 rules at each server, in a 2 hour training window immediately preceding the trial. We used 
all available rules (i.e. both the Snort and Nebula Sets), and selected which rules to rate at each server by ranking them based on their total number of alerts during the trial's training period at the server, and then rating the appropriate number of top ranked rules. For each number of rated rules, we then measured the average true positive and false positive rates when between 1 and 20 ROCK recommendations were used at each server. Figure 8 illustrates the curves for 5, 10, and 15 rated rules, with each curve showing the false positive, true positive pairs for all possible numbers of recommended rules. Figure 9 plots an estimate of the area under each of these curves. These graphs demonstrate that as users score more rules, ROCK recommendations provide them a better trade off between True Positive Rate and False Positive Rate.

The sharp elbow on the left of Figure 9 demonstrates that in our experiments, ROCK users needed only to rate a small number (under 5) of the most frequently matched rules at their IDSes to gain most of the benefits of ROCK recommendations. This represents a significant decrease from the number of rules a network operator working independently would need to analyze to tune an IDS. ROCK's effectiveness with limited operator input is also beneficial from a privacy perspective. Network operators concerned about revealing sensitive information when they collaborate can benefit from ROCK even if they only share a small number of vetted rule ratings.

We believe these results would generalize to production network IDSes and a live deployment of ROCK. With greater traffic diversity, a larger percentage of the available rule set may match traffic, suggesting that the curve of Figure 9 may stretch out further. However, our scenario only included 5 collaborators, far fewer than would participate in a live deployment of ROCK. With large numbers of collaborators, many collaborative filtering systems applied to other domains are able to make accurate recommendations using only a small number of ratings from each user [16]. Thus, overall a larger scale deployment of ROCK should still enable a significant reduction of individual IDS operator workload.

\section{Simulation Evaluation}

In this section, we evaluate ROCK in large scale statistical simulations. ROCK is designed to be generalizable, and our goal in this Section is to study that attribute. ROCK does not analyze the content of rules, or depend on the implementational details of IDS deployments. However, the amount of correlation between rules and networks does affect ROCK, and applying ROCK to different types of rules and IDSes may result in scenarios where these factors differ. To directly study how these factors impact ROCK, and thus better understand ROCK's generalizability, 


\begin{tabular}{|c|c|c|}
\hline Parameter & Meaning & Default Value \\
\hline$N$ & \# of networks & 100 \\
\hline$F$ & \# of flows & 10,000 \\
\hline$M$ & \% of flows malicious & $10 \%$ \\
\hline$G$ & \# networks per correlated group & 10 \\
\hline$R$ & \# of rules & 1000 \\
\hline$T$ & \# of correlated groups for each rule & 5 \\
\hline$S$ & \# of rules rated at each network & 20 \\
\hline
\end{tabular}

Table 6: Simulation parameters.

we model rules and IDS deployments using stochastic functions with parameters that control:

- rule effectiveness, or the likelihood of each rule generating a false alert or a true positive;

- correlation, between groups of rules, groups of IDS deployments, and sets of rules and IDS deployments;

- and user participation, or the number of rules rated by each user.

Table 6 summarizes the parameters of our simulation, their meanings, and their default values when not the independent variable in an experiment. We simulate a scenario with $N$ networks and IDS deployments, where each IDS observes $F$ distinct flows, $M$ percent of which are malicious. Each network belongs to a correlated group, which models a set of networks that observe similar levels of rule effectiveness. We assign each network to exactly one correlated group by generating $\frac{N}{G}$ correlated groups, and then selecting $G$ networks for each correlated group, uniformly at random and without repetition. There are $R$ total rules, and each rule matches traffic flows at all networks that belong to one of $T$ randomly selected correlated groups. A rule matches flows based on two parameters: its true positive rate, or its chance to match a malicious traffic flow; and its false positive rate, or its chance to match a non-malicious traffic flow. We assign a true positive rate to each rule $r$ in two stages that induce both network-dependent rule effectiveness and inter-network correlations. First, we draw a global component global $_{r}$ from the uniform $0.0-0.1$ distribution. Second, for each correlated group $g$ that the rule belongs to, we draw a group modifier modifier m $_{r, g}$ component from the $-0.01-0.01$ range. Finally, for all networks that belong to correlated group $g$, the rule is assigned a true positive rate of global $_{r}+$ modifier $_{r, g}$. We assign a false 


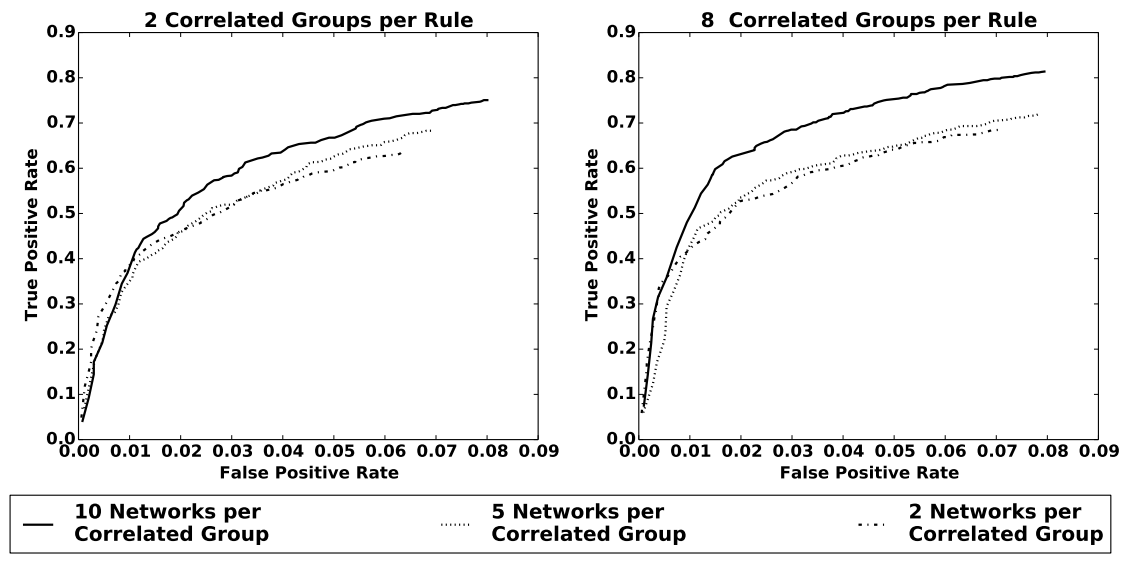

Figure 10: True and false positive rates of rule sets as the number of ROCK recommended rules increased, and the correlation between networks and rules varied.

positive rate to each rule using the same process, but with the global component drawn from the uniform $0.0-0.01$ distribution and the group modifier component drawn from the $-0.001-0.001$ distribution. Our choice of global rule parameters assumes that rules are generally effective, with false positive rates an order of magnitude lower than true positive rates, on average.

We divide each run of the simulator into two stages. First, a training stage where for each network we rate $S$ randomly selected rules by measuring their accuracy over the first half of the network's flows. We submit these ratings to ROCK, then in a testing stage we measure the effectiveness of rule sets selected using both local ratings and ROCK recommendations. Our goal in these experiments is not to measure the absolute effectiveness of ROCK, but rather to examine trends in recommendation quality as environmental factors that are likely change with deployment scale vary.

An important environmental factor to ROCK is the amount of correlation between rules and networks. We evaluated the quality of ROCK recommendations as the correlation varied via the parameters for the number of networks per correlated group and the number of correlated groups per rules. Figure 10 shows the effects of these parameters on true and false positive rates in a scenario where each network rated 20 rules during local training and deployed between 1 and 1000 ROCK recommended rules during testing. The depicted rates were averaged over all simulated networks in each run, and 100 runs of the simulator.

There are several important trends in this data that reveal the effects of scale and inter-network correlation on ROCK. First, as $G$, the number of networks per correlated groups increased, ROCK recommended better rules sooner. This trend 


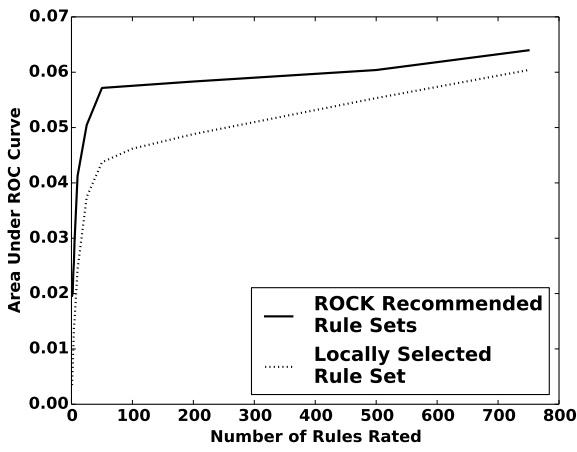

Figure 11: Area under false positive vs true positive curves as number of rules rated by each network increased.

demonstrates that ROCK is both sensitive to correlation, and likely to be increasingly effective as scale grows. $G$ represents an increase in number of networks that each individual user is similar to, which is likely to occur as the number of ROCK users grows.

ROCK also recommended rules more effectively as $T$ increased, demonstrating that ROCK's recommendations to a network are more sensitive to the observations of a small numbers of similar networks than observations from large numbers of dissimilar networks. As $T$ increases, a larger set of rules matches traffic at each correlated group of networks. In turn, these networks share ratings for a larger number of rules, which lead to improved ROCK recommendations. However, as $T$ increases, the correlation between pairs of networks not in a correlated group decreases: as a rule is observed at more correlated groups, which contain nonoverlapping sets of networks, more pairs of networks will observe different true and false positive rates from the rule. ROCK's greater sensitivity to similar networks than dissimilar network suggests that it would be able to leverage the diverse data obtained from large scale deployment.

We also observed that the quality of the top ROCK recommendations were not sensitive to either of these parameters for the range of values tested. In all cases, the top 20 recommended rules were highly effective, in terms of true positive rate vs false positive rate. This adds support to our hypothesis that ROCK is likely to provide benefit to network operators in a diverse range of scenarios.

We also measured how rating different numbers of rules affected the true and false positive rates of both ROCK and locally selected rule sets. Figure 11 plots the number of rules rated at each network vs the estimated area under the true positive vs false positive rate curves of rule sets built using increasing numbers of ROCK recommendations or locally rated rules, with all other parameters set 
to the values from Table 6. The increased values for ROCK recommended rule sets indicate that in most cases, the top $r$ ROCK recommended rules provided a better true positive to false positive ratio than the $r$ top locally rated rules. The operators of our simulated network would need to rate over 500 rules each to be able to select rules as effectively as they could by each providing ROCK with only 50 ratings, highlighting ROCK's usefulness as a tool to amplify the benefit of operator knowledge for optimizing local defense.

This result also demonstrates the benefit of ROCK for proactive defense. The overall effectiveness of the local rule selection technique did not reach that of the ROCK rule selection technique, as every rule did not match traffic at every IDS, preventing many IDSes from rating some of the rules. We observed this effect in our experiments with live traffic as well, and believe that it would be prevalent in any large scale deployment scenario.

\section{Discussion}

Based on our experimental results, ROCK would offer IDS operators many benefits as a production system; given the scale of existing recommender systems [36, 19], we believe ROCK as a web service or automated rule repository is within reach. In this section, we discuss areas of future research that we believe would prove useful in such production systems.

\subsection{Privacy}

Privacy is a critical concern for many network operators. However, collaboration requires data sharing. ROCK is designed to be a centralized platform, and does not share one user's data with other users. Thus, the primary privacy concern for ROCK users is whether they trust the party running the ROCK service. In practice, many IDS operators are willing to share information with trusted parties such as DShield [2], Sourcefire [5], the Emerging Threats Repository [3], and many antivirus companies. Deploying ROCK as a service run by such an organization would mitigate the concerns of many IDS operators.

There are also technical solutions that can be used to increase privacy, such as differential privacy, which provides provable statistical guarantees on the privacy of randomized functions to query aggregate data. Furthermore, it has already been applied to the domain of collaboratively analyzing statistical network data while maintaining privacy [29]. By implementing a differential privacy based approach for the sharing of rule effectiveness measurements, ROCK could provide IDS operators with both statistical guarantees of privacy, and the benefits of collaborative rule recommendations. Other work has also investigating general privacy preserving collaborative filtering techniques [11] that could be applied to ROCK. 

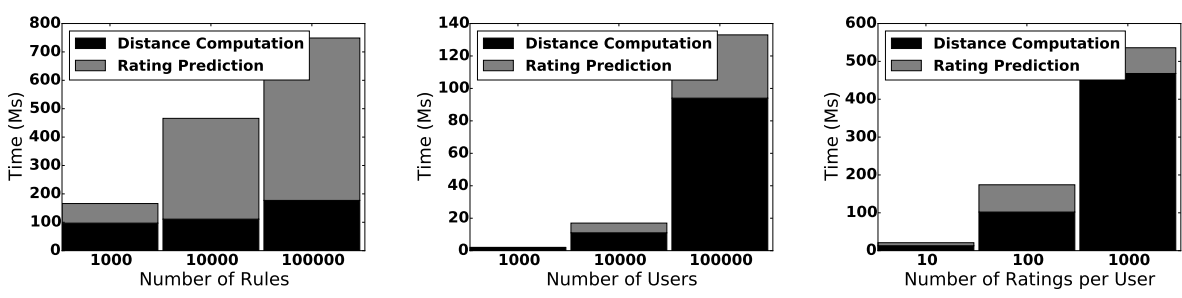

Figure 12: Computation time benchmarks as the number of rules, users, and rating provided by users grow, in milliseconds.

\subsection{Online Rule Updates}

Our current ROCK prototype operates asynchronously from IDS deployments: IDS operators submit rule ratings to ROCK, and request recommendations for new rules. After ROCK provides the recommendations, the operators can deploy the rules onto their IDSes however they wish.

Some IDSes, including Snort, allow the active rule set to be changed while the IDS remains online. ${ }^{3}$ ROCK could leverage this functionality to automatically update an IDSes rule set using a simple pull based model where each Operator runs a script that periodically:

1. Queries ROCK for the top X most recommended rules.

2. Compiles a new rule set consisting of the X ROCK recommended rules and any operator selected rules.

3. Signals the local Snort deployment to load the updated rule set.

This automation would allow an IDS deployment to rapidly adjust as collaborators share new information.

\subsection{Processing Costs}

To evaluate the feasibility of implementing ROCK as a centralized service, we also implemented the inner loop code of ROCK in $\mathrm{C}++$, which consists of computing all similarity and rule rating estimates for one user. This represents the most that ROCK would need to compute for a single user's recommendation request. Figure 12 shows the amount of time, in milliseconds, required to do this computation for a single user as the number of rules, users, and ratings provided by each user vary. The bars are split into time spent computing the similarities between users, and computing the predicted ratings for each rule. We used a baseline

\footnotetext{
${ }^{3}$ Snort allows this if compiled with the /emph-enable-reload option.
} 
of 10000 users, 1000 rules, and 100 ratings submitted per user. These baselines reflect a realistic scenario where there are more users than rules, and each user submits ratings for a fraction of the available rules. The benchmark was run on a single core of an Intel i7 CPU, and could easily be parallelized for multiple users (i.e. by computing each user's request on a different processor).

The most significant cost was due to distance computation, which grew linearly with the number of users and the number of ratings per user. Distance computation cost grew sublinearly with the number of rules, due to an efficient implementation of the Quadratic Chi distance metric [26]. The cost of predicting ratings also grew linearly with the number of users. However, it grew sublinearly with the number of rules because the rating prediction algorithm only takes into account pairs of users that have submitted ratings for one or more of the same rules, and increasing only the number of rules reduces the number of overlapping pairs of users.

These results demonstrate that a centralized ROCK implementation could easily provide recommendations for a large rule repository. For example, when we ran ROCK at the scale of the very popular Emerging Threats Repository (with 200, 000 users, 20, 000 rules, and 100 input ratings from each user), ROCK computed all the recommendations for each user in approximately 15 seconds. Based on these results, a 16 core server could provide each of the 200,000 users with updated ratings approximately once per day. There are also many optimizations that could be made once deployed - for example, similarities between IDSes that share no overlapping rules may not be necessary to compute.

\section{Conclusion}

With the ever increasing number of Internet based threats, rules, and rule generation techniques, network operators face a challenging knowledge building problem in selecting appropriate rules to deploy on their IDSes. ROCK, a system motivated by human-driven collaboration and based on widely successful collaborative filtering algorithms, solves this problem by recommending rules to users. IDS operators provide rule scores to ROCK; in turn, they receive recommendations about other rules that ROCK predicts will be effective on their IDSes. In a deployment where ROCK optimized IDS deployments for shellcode detection, we found that adding only 5 ROCK recommended rules to an IDS's rule set increased its detection rate by up to $150 \%$, without significantly affecting its usability. In our experiments, users could obtain most of the benefits of ROCK by rating only a small number of the rules that most frequently matched traffic at their IDS. Further, we found that ROCK was effective at recommending rules from rule generation techniques, even if users had never tested the rules on their IDSes, overcoming a barrier limiting widespread use of automatically generated rules and facilitating evolvable network 
defense. Using a statistical simulation, we found that ROCK's performance increased with scale and adapted well to varying levels of correlation. Our results suggest that ROCK would translate well to a deployed system, and motivate further research on improving local security with automated collaborative techniques.

\section{Acknowledgments}

This material is based upon work supported by the National Science Foundation Graduate Research Fellowship under Grant No. 1406177 and Grant No. 1406225. Any opinions, findings, and conclusions or recommendations expressed in this material are those of the author(s) and do not necessarily reflect the views of the National Science Foundation.

[1] Eu cybersecurity plan to protect internet and online freedom and opportunity. http://ec.europa.eu/digital-agenda/en/news/ eu-cybersecurity-plan-protect-open-internet-andonline-freedom-and-opportunity-cyber-security, 2013.

[2] Dshield.org. http://www.dshield.org/, 2015.

[3] Emerging threats repository. www . emergingthreats . net, 2015.

[4] Microsoft security intelligence report 15 . http://download. microsoft. com/download/5/0/3/50310CCE-8AF5-4FB483E2-03F1DA92F33C/Microsoft_Security_Intelligence_ Report_Volume_15_English.pdf, 2015.

[5] The snort rule repository. https://www. snort.org/downloads/ \#rule-downloads, 2015.

[6] Suricata: Open Source IDS / IPS / NSM engine. http://suricataids. org, 2015.

[7] Gediminas Adomavicius and Alexander Tuzhilin. Toward the next generation of recommender systems: A survey of the state-of-the-art and possible extensions. Knowledge and Data Engineering, IEEE Transactions on, 17(6):734749, 2005.

[8] K.G. Anagnostakis, S. Sidiroglou, P. Akritidis, K. Xinidis, E. Markatos, and A.D. Keromytis. Detecting targeted attacks using shadow honeypots. In Proceedings of the 14th USENIX security symposium, volume 144, 2005.

[9] P Baecher and M Koetter. Libemu - x86 shellcode emulation. Iibemu. carnivore.it/, 2009.

[10] Tim Bass. Intrusion detection systems and multisensor data fusion. Сотmunications of the ACM, 43(4):99-105, 2000.

[11] John Canny. Collaborative filtering with privacy. In Security and Privacy, 2002. Proceedings. 2002 IEEE Symposium on, pages 45-57. IEEE, 2002. 
[12] Niall Fitzgibbon and Mike Wood. Conficker. c: A technical analysis. SophosLabs, Sophon Inc, 2009.

[13] G. Giacinto, F. Roli, and L. Didaci. Fusion of multiple classifiers for intrusion dection in computer networks. Pattern Recognition Letters, 24(12):17951803, 2003.

[14] David Goldberg, David Nichols, Brian M Oki, and Douglas Terry. Using collaborative filtering to weave an information tapestry. Communications of the ACM, 35(12):61-70, 1992.

[15] Markus Koetter. Dionaea low interaction honeypot. http://dionaea. carnivore.it/, 2009.

[16] Yehuda Koren. The bellkor solution to the netflix grand prize. Netflix prize documentation, 2009.

[17] C. Kreibich and J. Crowcroft. Honeycomb: creating intrusion detection signatures using honeypots. ACM SIGCOMM Computer Communication Review, 34(1):51-56, 2004.

[18] S. C. Lee and D. V. Heinbuch. Training a neural-network based intrusion detector to recognize novel attacks. IEEE Trans. Systems, Man and Cybernetics A, 31:294-299, 2001.

[19] Greg Linden, Brent Smith, and Jeremy York. Amazon. com recommendations: Item-to-item collaborative filtering. Internet Computing, IEEE, 7(1):76-80, 2003.

[20] H. Ling and K. Okada. Diffusion distance for histogram comparison. In Computer Vision and Pattern Recognition, 2006 IEEE Computer Society Conference on, volume 1, pages 246-253. IEEE, 2006.

[21] H. Ling and K. Okada. An efficient earth mover's distance algorithm for robust histogram comparison. Pattern Analysis and Machine Intelligence, IEEE Transactions on, 29(5):840-853, 2007.

[22] M. Locasto, K. Wang, A. Keromytis, and S. Stolfo. Flips: Hybrid adaptive intrusion prevention. In RAID, pages 82-101. Springer, 2006.

[23] J. Newsome, B. Karp, and D. Song. Polygraph: Automatically generating signatures for polymorphic worms. In Security and Privacy, 2005 IEEE Symposium on, pages 226-241. IEEE, 2005.

[24] Office of the Press Secretary. Fact sheet: Executive order promoting private sector cybersecurity information sharing. https: //www.whitehouse.gov/the-press-office/2015/02/ 12 / fact-sheet-executive-order-promoting-privatesector-cybersecurity-inform, 2015.

[25] V. Paxson. Bro: A system for detecting network intruders in real-time. Computer networks, 31(23):2435-2463, 1999. 
[26] O. Pele and M. Werman. The quadratic-chi histogram distance family. Computer Vision-ECCV 2010, pages 749-762, 2010.

[27] Georgios Portokalidis, Asia Slowinska, and Herbert Bos. Argos: an emulator for fingerprinting zero-day attacks for advertised honeypots with automatic signature generation. In ACM SIGOPS Operating Systems Review, volume 40, pages 15-27. ACM, 2006.

[28] M Zubair Rafique and Juan Caballero. Firma: Malware clustering and network signature generation with mixed network behaviors. In Research in Attacks, Intrusions, and Defenses, pages 144-163. Springer, 2013.

[29] J. Reed, A.J. Aviv, D. Wagner, A. Haeberlen, B.C. Pierce, and J.M. Smith. Differential privacy for collaborative security. In Proceedings of the Third European Workshop on System Security, pages 1-7. ACM, 2010.

[30] Paul Resnick, Neophytos Iacovou, Mitesh Suchak, Peter Bergstrom, and John Riedl. Grouplens: an open architecture for collaborative filtering of netnews. In Proceedings of the 1994 ACM conference on Computer supported cooperative work, pages 175-186. ACM, 1994.

[31] M. Roesch et al. Snort-lightweight intrusion detection for networks. In Proceedings of the 13th USENIX conference on System administration, pages 229-238. Seattle, Washington, 1999.

[32] S. Snapp, J. Brentano, G. Dias, T. Goan, L. Heberlein, C. Ho, K. Levitt, B. Mukherjee, S. Smaha, T. Grance, D. Teal, and D. Mansur. Dids (distributed intrusion detection system) - motivation, architecture and an early prototype. In Proc. National Computer Security Conference, 1991.

[33] Joel Sommers, Vinod Yegneswaran, and Paul Barford. Recent advances in network intrusion detection system tuning. In Information Sciences and Systems, 2006 40th Annual Conference on, pages 1490-1495. IEEE, 2006.

[34] John Sonchack, Adam J.Aviv, and Jonathan M. Smith. Bridging the data gap: Data related challenges in evaluating large scale collaborative security systems. In Proceedings of the 6th CSET Workshop, 2013.

[35] S. Sturges. Using Perfmon and Performance Profiling to Tune Snort Preprocessors and Rules. Sourcefire, 2009.

[36] Xiaoyuan Su and Taghi M Khoshgoftaar. A survey of collaborative filtering techniques. Advances in artificial intelligence, 2009:4, 2009.

[37] G. Vasiliadis, S. Antonatos amd M. Polychronakis, E. Markatos, and S. Ioannidis. Gnort: High performance network intrusion detection using graphics processors. In Proc. 11th International Symp. on Recent Advances in Intrusion Detecion. Springer-Verlag, 2008.

[38] K. Wang, G. Cretu, and S. Stolfo. Anomalous payload-based worm detection and signature generation. In RAID, pages 227-246. Springer, 2006. 
[39] Tillmann Werner, Christoph Fuchs, Elmar Gerhards-Padilla, and Peter Martini. Nebula-generating syntactical network intrusion signatures. In Malicious and Unwanted Software (MALWARE), 2009 4th International Conference on, pages 31-38. IEEE, 2009.

[40] V. Yegneswaran, P. Barford, and J. Sha. Global intrusion detection in the domino overlay system. In Proc. NDSS, 2004.

[41] V. Yegneswaran, J.T. Giffin, P. Barford, and S. Jha. An architecture for generating semantics-aware signatures. In Proceedings of the 14th USENIX security symposium, volume 112. Baltimore, MD, USA, 2005.

[42] J. Zhang, P. Porras, and J. Ullrich. Highly predictive blacklisting. In Proceedings of the 17th conference on Security symposium, pages 107-122. USENIX Association, 2008.

[43] J. Zhang, P. Porras, and J. Ullrich. Gaussian process learning for cyber-attack early warning. Statistical Analysis and Data Mining, 2010. 